\title{
On the structure of sets with small doubling property on the plane (I)
}

\author{
by
}

\author{
Yonutz Stanchescu (Tel-Aviv)
}

Let $K$ be a finite set of lattice points in a plane. We prove that if $|K|$ is sufficiently large and $|K+K|<(4-2 / s)|K|-(2 s-1)$, then there exist $s-1$ parallel lines which cover $K$. We also obtain some more precise structure theorems for the cases $s=3$ and $s=4$.

1. Introduction, notation and results. Let $\mathbb{R}^{n}$ be the $n$-dimensional Euclidean space and $\mathbb{Z}^{n}$ the additive group of integral vectors in $\mathbb{R}^{n}$. Given a finite set $M \subseteq \mathbb{R}^{n}$, the number of its elements will be denoted by $|M|=m$. We denote by $M+N$ the algebraic sum of two finite sets and $2 M=M+M$ is called the sum set of $M$. Let $M 2$ be the set $\{2 x: x \in M\}$. The convex hull of $M$ is denoted by $\operatorname{conv}(M)$. Vectors will be written in the form $\left(\kappa_{1}, \ldots, \kappa_{n}\right)$, where $\kappa_{i}, 1 \leq i \leq n$, are the coordinates of the vector.

Let $G_{1}$ and $G_{2}$ be commutative groups, $A_{1} \subseteq G_{1}, A_{2} \subseteq G_{2}$. We say that a mapping $\phi: A_{1} \rightarrow A_{2}$ is a homomorphism of order 2 in the sense of Freiman, or an $F_{2}$-homomorphism for short, if for all $x_{1}, x_{2}, y_{1}, y_{2} \in A_{1}$ (not necessarily distinct) the equation

$$
\text { (i) } x_{1}+x_{2}=y_{1}+y_{2}
$$

implies

$$
\text { (ii) } \phi\left(x_{1}\right)+\phi\left(x_{2}\right)=\phi\left(y_{1}\right)+\phi\left(y_{2}\right) \text {. }
$$

We call $\phi$ an $F_{2}$-isomorphism if it is one-to-one and its inverse is also a homomorphism, that is, (ii) holds if and only if (i) does.

Let $M_{1}$ and $M_{2}$ be finite subsets of $\mathbb{R}^{n}$. We say that $M_{1}$ is isomorphic to $M_{2}$ if there is an affine isomorphism $L: \mathbb{R}^{n} \rightarrow \mathbb{R}^{n}$ such that $L\left(M_{1}\right)=M_{2}$. Obviously, $M_{1}$ is then $F_{2}$-isomorphic to $M_{2}$.

A direct problem in set addition theory asks what can be said about $|M+M|$ for a given set $M$. Clearly

1991 Mathematics Subject Classification: 11P99, $11 B 99$. 


$$
2|M|-1 \leq|M+M| \leq \frac{1}{2}|M|(|M|+1)
$$

and if $M$ is an arithmetic progression, then $|M+M|=2|M|-1$. The inverse problem determines the structure of $M$ if $|M+M|$ is given or if $|M+M|<c_{0}|M|$ with $c_{0}$ a positive number.

We can easily see that if $|M+M|=2|M|-1$, then $M$ is an arithmetic progression. If we choose larger values for $|M+M|$ the problem ceases to be trivial. The fundamental theorem of G. A. Freiman [F1], p. 54, gives the structure of finite sets $M$ for the case $|M+M|<c|M|$, where $c$ is any given positive number. The theorem was proved using analytical methods of number theory and a modification of the method of trigonometric sums. An improved version of the proof is presented in [F3], and Yuri Bilu [B] studied the case when $c$ is a slowly growing function of $|M|$. I. Z. Ruzsa [R] recently gave a new and shorter proof of the main theorem together with an important generalization to the case of different summands $K+M$.

However, when the values of the constant are small, elementary methods yield sharper results. Two cases have been studied by G. A. Freiman [F1], pp. 11, 28.

TheOREM 1.1. Let $K \subseteq \mathbb{Z}$ be a finite set of integers. If $|K+K|=$ $2|K|-1+b$ where $0 \leq b \leq k-3$, then $K$ is contained in an arithmetic progression of length $k+b=|K+K|-k+1$, where $k=|K|$.

TheOREM 1.2. Let $K \subseteq \mathbb{Z}^{2}$ be a finite set which is not a subset of any straight line. Suppose $|K+K|<\frac{10}{3}|K|-5$ and $k=|K| \geq 11$. Then $K$ is contained in a set which is isomorphic to $K_{0}=\left\{(0,0),(1,0), \ldots,\left(l_{1}-\right.\right.$ $\left.1,0) ;(0,1), \ldots,\left(l_{2}-1,1\right)\right\}$ where $l_{1}, l_{2} \geq 1$ and $l_{1}+l_{2}=|K+K|-2 k+3$.

Using the same ideas we will prove in Section 2:

Theorem A. Let $K$ be a finite subset of $\mathbb{Z}^{2}$. If $k=|K| \geq k_{0}(s)$ is sufficiently large and if

$$
|K+K|<(4-2 / s)|K|-(2 s-1) \quad(s>1)
$$

then there exist $s-1$ parallel lines which cover the set $K$.

The above constant $k_{0}(s)$ is effective and an examination of the proof will show that it is of order $O\left(s^{3}\right)$. Example A, which ends Section 2, shows that Theorem A cannot be improved by increasing the upper bound $(4-2 / s) k-(2 s-1)$ of $|2 K|$.

In Section 3 we formulate and prove a sharpened version of Theorem 1.2. We assume that $K$ lies on two parallel lines and prove that Theorem 1.2 is true even if we replace $|2 K|<\frac{10}{3} k-5$ by $|2 K|<4 k-6$. This is the maximum possible value for the upper bound of $|2 K|$.

Theorem B. Let $K$ be a finite subset of $\mathbb{Z}^{2}$ which lies on two parallel lines, but is not contained in a single line. Suppose $|K+K|<4|K|-6$. 
Then $K$ is contained in a set which is isomorphic to

$$
K_{0}=\left\{(0,0),(1,0), \ldots,\left(l_{1}-1,0\right) ;(0,1),(1,1), \ldots,\left(l_{2}-1,1\right)\right\},
$$

where $l_{1}, l_{2} \geq 1$ and $l_{1}+l_{2}=|K+K|-2 k+3$.

As usual, the solution of an inverse problem allows us to obtain nontrivial lower bounds for $|K+K|$, thus solving at the same time a direct additive problem (see Theorem B* in Section 3). We also provide two Examples B.1 and B.2 showing that Theorem B cannot be sharpened by increasing the upper bound for $|K+K|$ or by reducing the quantity $l_{1}+l_{2}$.

Moreover, as in the cases $s=2$ (Theorem 1.1) and $s=3$ (Theorem 1.2), we prove in Section 4 a more precise structure theorem for $s=4$ :

Theorem C. Let $K$ be a finite subset of $\mathbb{Z}^{2}$, not contained in any two parallel lines. Suppose $|K+K|<3.5|K|-7$ and $k=|K| \geq k_{0}$. Then $K$ is contained in a set isomorphic to

$$
\begin{aligned}
& K_{0}=\left\{(0,0),(1,0), \ldots,\left(l_{1}-1,0\right) ;(0,1),\right.(1,1), \ldots,\left(l_{2}-1,1\right) ; \\
&\left.(0,2),(1,2), \ldots,\left(l_{3}-1,2\right)\right\},
\end{aligned}
$$

where $l_{1}, l_{2}, l_{3} \geq 1$ and $\max \left(l_{1}, l_{2}, l_{3}\right) \leq \frac{1}{2}(|K+K|-2 k+3)$.

The last inequality shows that if $|K+K|<3.5|K|-7$ and $k \geq k_{0}$, then

$$
|K+K| \geq(2|K|-1)+2\left(\max \left(l_{1}, l_{2}, l_{3}\right)-1\right) .
$$

As can be easily seen by considering Example C, Section 4, the lower bound in (1.4) is best possible. Moreover, we will show that $k_{0}=k_{0}(4)=1344$.

2. On the structure of $K \subseteq \mathbb{Z}^{2}$ with $|K+K|<(4-2 / s)|K|-(2 s-1)$. In this section, we prove Theorem $\mathrm{A}$. We need the following lemma:

Lemma 2.1. Let $s>1$ be a natural number. There exists a positive constant $\delta=\delta(s)$ such that if $K \subseteq \mathbb{Z}^{2}$ is a finite set of lattice points with

$$
|K+K|<(4-2 / s)|K|-(2 s-1),
$$

then there exists a line $\ell$ in $\mathbb{R}^{2}$ such that

$$
|\ell \cap K| \geq \delta|K| \text {. }
$$

Lemma 2.1 is a particular case of Lemma 2.12 of [F1], p. 57, and we will give here an independent proof which improves the value of $\delta(s)$. For a proof of the general case, see also [B], pp. 11-17.

Before the proof of the lemma, let us notice the following three inequalities. Suppose that $K$ is decomposable into $r$ subsets $K_{1}, \ldots, K_{r}$ which lie on $r$ parallel lines properly ordered (there exist real numbers $t_{1}<\ldots<t_{r}$ such that, after a coordinate change, the line containing $K_{i}$ is defined by 
the equation $x_{2}=t_{i}$ ). If $k_{i}=\left|K_{i}\right| \geq 1$ for every $i=1, \ldots, r$, then we can easily prove that (see $[\mathrm{F} 1]$, p. 25)

$$
\begin{array}{ll}
\text { ( } \alpha) & |K+K| \geq 4 k-\left(k_{1}+k_{r}\right)-(2 r-1), \\
(\beta) & |K+K| \geq 2 k+\left(k_{1}+k_{r}-2\right)(r-1)-1 .
\end{array}
$$

Multiplying inequality $(\alpha)$ by $r-1$ and adding it to $(\beta)$ we get

$$
|K+K| \geq(4-2 / r) k-(2 r-1) .
$$

Lemma 2.1 is an easy consequence of Lemma 2.2 below.

Lemma 2.2. Suppose that $K \subseteq \mathbb{Z}^{2}$ satisfies the assumption of Lemma 2.1 and let $\delta_{0}(s)=1 /(4 s-4)$. Then either

(a) there exists a line $\ell$ such that $|\ell \cap K| \geq \delta_{0}(s)|K|$, or

(b) there exists a proper subset $K_{0} \subseteq K$ such that

$$
0<|K|-\left|K_{0}\right| \leq 2 s-2, \quad\left|K_{0}+K_{0}\right| \leq|K+K|-4\left(|K|-\left|K_{0}\right|\right) .
$$

Pro of. Let $D=\operatorname{conv}(K)$ be the convex hull of $K$. Then $D$ is a polygon whose vertices are all contained in $K$. We consider an arbitrary vertex $V_{1}$ together with two edges of the boundary of $D$ which intersect at $V_{1}$ and two additional points $V_{2}$ and $V_{3}$ on these edges. Here we choose $V_{2}, V_{3} \in K$ so that there is no other point of $K$ between $V_{1}$ and $V_{2}$ or between $V_{1}$ and $V_{3}$.

We will examine four cases and prove that (a) or (b) holds.

CASE A: The set $K$ does not lie in the lattice generated by the points $V_{1}, V_{2}, V_{3}$. We show that (b) holds. There is a vector $v \in K$,

$$
v=\alpha\left(V_{2}-V_{1}\right)+\beta\left(V_{3}-V_{1}\right),
$$

for which at least one of the coordinates $\alpha$ and $\beta$ is not a positive integer. Select $v$ in such a way that there is no other vector $v^{\prime}$ with coordinates satisfying $\alpha^{\prime} \leq \alpha$ and $\beta^{\prime} \leq \beta$. Then $2 V_{1}, V_{1}+V_{2}, V_{1}+V_{3}$ and $V_{1}+v$ do not belong to the set $2\left(K \backslash\left\{V_{1}\right\}\right)$. Therefore

$$
\left|2\left(K \backslash\left\{V_{1}\right\}\right)\right| \leq|2 K|-4 .
$$

If the set $K$ lies in the lattice generated by $V_{1}, V_{2}, V_{3}$ it can be mapped, by a linear transformation, onto an isomorphic set which lies in the first quadrant where the points have nonnegative integer coordinates. We may suppose that $V_{1}=(0,0), V_{2}=(1,0)$ and $V_{3}=(0,1)$.

Case B: On the line $\left\{x_{2}=0\right\}$ defined by $(0,0)$ and $(1,0)$ we have at least $2 s-1$ points of $K$. We show that $K$ lies on no more than $s-1$ parallel lines.

Suppose that $K$ is decomposable into $r$ subsets $K_{1}, \ldots, K_{r}$ which lie on $r$ lines, parallel to the axis $\left\{x_{2}=0\right\}$. 
(i) If $s \leq r \leq k / s$ it follows from (2.2) that

$$
|2 K| \geq(4-2 / r) k-(2 r-1) \geq(4-2 / s) k-(2 s-1),
$$

which contradicts (1.2).

(ii) If $r>k / s$ we deduce from (2.1) that

$$
\begin{aligned}
|2 K| & \geq 2 k+\left(k_{1}+k_{r}-2\right)(r-1)-1 \geq 2 k+(2 s-2)(k / s-1)-1 \\
& =4 k-2 \frac{k}{s}-2 s+1=(4-2 / s) k-(2 s-1),
\end{aligned}
$$

which contradicts (1.2). Finally, we conclude that $K$ lies on no more than $s-1$ lines parallel to $\left\{x_{2}=0\right\}$. In this case, (a) is proved with $\delta_{0}=1 /(s-1)$.

CASE C: On the line $\left\{x_{2}=0\right\}$ we have no more than $2 s-2$ points of $K$ and these points are not an arithmetic progression. Suppose that $(c, 0)$ and $(d, 1)$ are points of $K$ with maximal abscissa on the lines $\left\{x_{2}=0\right\}$ and $\left\{x_{2}=1\right\}$, respectively. Let $[c, d)$ be the semi-line defined by these two points. Let $H$ be the convex body defined by $[c, d)$ and the two semi-axes $\left\{x_{1}=0\right\},\left\{x_{2}=0\right\}$, that is, the region defined by the inequalities

$$
x_{1} \geq 0, \quad x_{2} \geq 0, \quad x_{1} \leq c+(d-c) x_{2} .
$$

We will use the notation

$$
\begin{aligned}
K_{1} & =K \cap\left\{x_{2}=0\right\}, & K_{2} & =K \cap\left\{x_{2}=1\right\}, \\
k_{1} & =\left|K_{1}\right| \leq 2 s-2, & k_{2} & =\left|K_{2}\right| .
\end{aligned}
$$

Since $K_{1}$ is not an arithmetic progression, we thus have $c \geq 3,\left|K_{1}\right| \geq 3$, $\left|2 K_{1}\right| \geq 2 k_{1}$ and $\left|K_{1}+K_{2}\right| \geq k_{1}+k_{2}$. There are two cases to consider: $d>c$ or $d \leq c$.

(i) If $d>c$ and $k_{2}<k_{1}$, then the removal of $K_{1}$ from $K$ reduces the cardinality of $2 K$ by at least $4 k_{1}$ since

$$
\left|2\left(K \backslash K_{1}\right)\right| \leq|2 K|-\left|2 K_{1}\right|-\left|K_{1}+(0,1)\right|-\left|K_{1}+(d, 1)\right| \leq|2 K|-4 k_{1} .
$$

If $k_{2} \geq k_{1}$, then the removal of $K_{1}$ from $K$ reduces the cardinality of $2 K$ by at least $4 k_{1}$ since

$$
\begin{aligned}
\left|2\left(K \backslash K_{1}\right)\right| & \leq|2 K|-\left|2 K_{1}\right|-\left|K_{1}+K_{2}\right| \leq|2 K|-\left(2 k_{1}+k_{1}+k_{2}\right) \\
& \leq|2 K|-4 k_{1} .
\end{aligned}
$$

We conclude that (b) holds if $d>c$.

(ii) If $d \leq c$ and $K$ is not included in $H$, then the removal of $(c, 0)$ reduces the cardinality of $2 K$ by at least 4 :

$$
|2(K \backslash(c, 0))| \leq|2 K|-4 .
$$

(Indeed, we use inequality $(2.3)$ with $V_{1}=(c, 0)$.) 
If $d \leq c$ and $K$ is included in $H$, then we apply Freiman's Theorem 1.1. First, we suppose $d \geq 3$, otherwise it follows that

$K$ is covered by four lines parallel to $\left\{x_{2}=0\right\}$.

(Indeed, if $0 \leq d \leq 1$, then $K$ is covered by $\left\{x_{2}=0\right\}$ and $\left\{x_{2}=1\right\}$. If $d=2$, then $K$ is covered by $\left\{x_{2}=0\right\},\left\{x_{2}=1\right\},\left\{x_{2}=2\right\}$ and $\left\{x_{2}=3\right\}$. Note that we used $d \leq 2<c$.)

If $c=\max K_{1} \leq 2 k_{1}-3$, then using the inequality $k_{1} \leq 2 s-2$ we get $c \leq 4 s-7$; thus

$K$ lies on no more than $4 s-6$ lines parallel to $\left\{x_{1}=0\right\}$.

If $c=\max K_{1}>2 k_{1}-3$, Freiman's Theorem implies that $\left|2 K_{1}\right| \geq 3 k_{1}-3$. If $k_{2} \geq 3$, then the removal of $K_{1}$ from $K$ reduces the cardinality of $2 K$ by at least $4 k_{1}$, since

$$
\begin{aligned}
\left|2\left(K \backslash K_{1}\right)\right| & \leq|2 K|-\left|2 K_{1}\right|-\left|K_{1}+K_{2}\right| \\
& \leq|2 K|-\left(3 k_{1}-3\right)-\left(k_{1}+3\right) \leq|2 K|-4 k_{1} .
\end{aligned}
$$

If on $\left\{x_{2}=1\right\}$ we have only two points $(0,1)$ and $(d, 1)$, then in the "general" case we still obtain $\left|K_{1}+K_{2}\right| \geq k_{1}+3$. More exactly, if $K_{1}$ is not equal to $L=\{(0,0),(d, 0),(2 d, 0), \ldots\} \cup\{(1,0),(1+d, 0),(1+2 d, 0), \ldots\}$, then

$$
\left|K_{1}+K_{2}\right| \geq k_{1}+3
$$

and (as above) the removal of $K_{1}$ from $K$ reduces the cardinality of $2 K$ by at least $4 k_{1}$; that is,

$$
\left|2\left(K \backslash K_{1}\right)\right| \leq|2 K|-4 k_{1} .
$$

If $K_{1}=L$, that is, $K_{1}$ is the union of two arithmetic progressions modulo $d$, with $d \geq 3$, then the removal of $(0,0)$ from $K$ reduces the cardinality of $2 K$ by at least 4 : the points $(0,0),(0,1),(1,0),(d, 0)$ are in $2 K$ but not in $2(K \backslash(0,0))$; therefore

$$
|2(K \backslash(0,0))| \leq|2 K|-4 .
$$

Before examining case D, let us make one further remark. Suppose that $k_{2}=2$ and $K$ does not lie on $\left\{x_{2}=0\right\} \cup\left\{x_{2}=1\right\}$. We show that the removal of $K_{1}$ from $K$ reduces the cardinality of $2 K$ by at least $4 k_{1}$ and therefore (2.10) is again true. Indeed, if $t>1$ is the smallest number such that $K_{3}=K \cap\left\{x_{2}=t\right\}$ satisfies $\left|K_{3}\right|=k_{3} \geq 1$, then

$$
\begin{aligned}
|2 K| & \geq\left|2\left(K \backslash K_{1}\right)\right|+\left|K_{1}+K_{1}\right|+\left|K_{1}+K_{2}\right|+\left(\left|K_{1}+K_{3}\right|-\left|2 K_{2}\right|\right) \\
& \geq\left|2\left(K \backslash K_{1}\right)\right|+\left(3 k_{1}-3\right)+\left(k_{1}+2\right)+\left(k_{1}+k_{3}-3\right) \\
& \geq\left|2\left(K \backslash K_{1}\right)\right|+4 k_{1}+\left(k_{1}+k_{3}-4\right) \geq\left|2\left(K \backslash K_{1}\right)\right|+4 k_{1} .
\end{aligned}
$$

CASE D: The points of $K_{1}=K \cap\left\{x_{2}=0\right\}$ form an arithmetic progression with no more than $2 s-2$ elements. Let $K_{1}=K \cap\left\{x_{2}=0\right\}=$ 
$\{(0,0),(1,0), \ldots,(c-1,0)\}$ with $c \leq 2 s-2$ and let $(d, 1)$ be the point of $K_{2}=K \cap\left\{x_{2}=1\right\}$ with maximal abscissa on the line $\left\{x_{2}=1\right\}$. As in case $\mathrm{C}$, we define the convex set $H$ by the inequalities

$$
x_{1} \geq 0, \quad x_{2} \geq 0, \quad x_{1} \leq(c-1)+(d-c+1) x_{2} .
$$

If $K$ is not included in $H$, then the removal of $(c-1,0)$ from $K$ reduces the cardinality of $2 K$ by at least 4 :

$$
|2(K \backslash(c-1,0))| \leq|2 K|-4 .
$$

If $K$ is included in $H$ and if $d \leq c-1$, then

$$
K \text { lies on no more than } 2 s-2 \text { lines parallel to }\left\{x_{1}=0\right\} \text {. }
$$

If $K \subseteq H, d \geq c+1$ and $K_{2} \neq\{(0,1),(d, 1)\}$, then $\left|K_{1}+K_{2}\right| \geq 2 k_{1}+1$. Thus, the removal of $K_{1}$ from $K$ reduces the cardinality of $2 K$ by at least $4 k_{1}$. Indeed,

$$
\begin{aligned}
\left|2\left(K \backslash K_{1}\right)\right| & \leq|2 K|-\left|2 K_{1}\right|-\left|K_{1}+K_{2}\right| \\
& \leq|2 K|-\left(2 k_{1}-1\right)-\left(2 k_{1}+1\right) \leq|2 K|-4 k_{1} .
\end{aligned}
$$

If $K \subseteq H, d \geq c+1$ and $K_{2}=\{(0,1),(d, 1)\}$, then the removal of $(0,0)$ from $K$ reduces the cardinality of $2 K$ by at least 4 : the points $(0,0),(0,1),(1,0),(d, 1)$ are all in $2 K$ but not in $2(K \backslash(0,0))$. Therefore

$$
|2(K \backslash(0,0))| \leq|2 K|-4 \text {. }
$$

If $K \subseteq H$ and $d=c$, then the set $K$ lies in the convex region defined by the inequalities

$$
x_{1} \geq 0, \quad x_{2} \geq 0, \quad x_{2} \geq x_{1}-(c-1) .
$$

Moreover, $c$ is bounded in terms of $s$ :

$$
1 \leq c \leq 2 s-2 \text {. }
$$

We may suppose that we have the same situation on the lines $\left\{x_{1}=0\right\}$, $\left\{x_{1}=1\right\}$, that is, $K$ lies in the convex region defined by the inequalities

$$
x_{2} \geq 0, \quad x_{1} \geq 0, \quad x_{1} \geq x_{2}-(e-1),
$$

with $1 \leq e \leq 2 s-2$. Note that $(c-1)+(e-1)+1 \leq 4 s-5$. We conclude that

$$
K \text { lies on no more than } 4 s-5 \text { lines parallel to }\left\{x_{2}=x_{1}\right\} \text {. }
$$

We may now complete the proof of Lemma 2.2 without difficulty. As in case B, if one of the cases (2.7), (2.8), (2.13), (2.16) holds, then we can easily find the number $\delta_{0}=\delta_{0}(s)$ claimed by (a). Actually, we may choose $\delta_{0}=\delta_{0}(s)=1 /(4 s-4)$. Otherwise we apply (2.3)-(2.6), (2.9)(2.12), (2.14), (2.15) and we prove (b). 
Pro of of Lemma 2.1. Let us say that the set $K$ is good if it satisfies condition (a). Let $K^{\prime}$ be a minimal subset of $K$ satisfying

$$
\left|K^{\prime}+K^{\prime}\right| \leq|K+K|-4\left(|K|-\left|K^{\prime}\right|\right)
$$

and

$$
\left|K^{\prime}\right| \geq \frac{k}{2 s}
$$

In order to prove Lemma 2.1 it is enough to show that $K^{\prime}$ is good. Suppose that $K^{\prime}$ is not good. Note that $K^{\prime}$ satisfies the small doubling property (1.2). By applying (a) and (b) there exists $K^{\prime \prime} \subseteq K^{\prime}$ satisfying

$$
\begin{aligned}
0<\left|K^{\prime}\right|-\left|K^{\prime \prime}\right| & \leq 2 s-2, \\
\left|K^{\prime \prime}+K^{\prime \prime}\right| & \leq\left|K^{\prime}+K^{\prime}\right|-4\left(\left|K^{\prime}\right|-\left|K^{\prime \prime}\right|\right) .
\end{aligned}
$$

Then by (2.17) and (2.20) we obtain

$$
\left|K^{\prime \prime}+K^{\prime \prime}\right| \leq|K+K|-4\left(|K|-\left|K^{\prime \prime}\right|\right) \text {. }
$$

Therefore we should have

$$
\left|K^{\prime \prime}\right|<\frac{k}{2 s},
$$

otherwise there would be a contradiction to the minimal choice of $K^{\prime}$. Now (2.18), (2.19) and (2.22) yield that

$$
\frac{k}{2 s} \leq\left|K^{\prime}\right|<\frac{k}{2 s}+2 s-2 .
$$

Combining this with (2.17) we get

$$
\left|K^{\prime}+K^{\prime}\right|<\left(4-\frac{2}{s}\right) k-(2 s-1)-4 k+4 \frac{k}{2 s}+4(2 s-2)=6 s-7,
$$

which is a contradiction if $k>s(6 s-6)$. (Indeed, $\left|K^{\prime}+K^{\prime}\right| \geq 2\left|K^{\prime}\right|-1 \geq$ $k / s-1>6 s-7$.)

We have proved that there is a good subset $K^{\prime} \subseteq K$ satisfying

$$
\left|K^{\prime}\right| \geq \frac{k}{2 s} \text {. }
$$

Then for some line $\ell$ we have

$$
\left|\ell \cap K^{\prime}\right| \geq \delta_{0}(s)\left|K^{\prime}\right|
$$

from which

$$
|\ell \cap K| \geq \frac{\delta_{0}(s)}{2 s}|K|=\delta(s)|K| \quad \text { with } \quad \delta(s)=\frac{1}{2 s} \delta_{0}(s) .
$$

Thus the assertion of Lemma 2.1 is proved for $|K|>s(6 s-s)$. Now assume that $|K| \leq s(6 s-6)$. For every line $\ell^{\prime}$ that contains at least two points of $K$ we may write

$$
\left|\ell^{\prime} \cap K\right| \geq 2 \geq \delta(s)|K|
$$


since $|K| \leq s(6 s-6) \leq 4 s(4 s-4)=2 / \delta(s)$. The proof of Lemma 2.1 is now complete.

REMARK. As we see from the proof of Lemma 2.1, we may choose the following value for the constant $\delta(s)$ :

$$
\delta=\delta(s)=\frac{1}{2 s(4 s-4)} \gg \frac{1}{s^{2}} .
$$

Proof of Theorem A. By Lemma 2.1, there exists a line $\ell$ in $\mathbb{R}^{2}$ such that $|\ell \cap K| \geq \delta k$, where $\delta=\delta(s)$ is given by (2.24). Let us cover $K$ by $r$ lines parallel to $\ell$. Using the inequality $|K+K|<(4-2 / s) k$ we get

and so

$$
r \delta k \leq r|\ell \cap K| \leq|K+K| \leq \frac{4 s-2}{s} k
$$

$$
r \leq \frac{4 s-2}{s \delta}
$$

If we suppose that $k \geq(4 s-2) / \delta$, then we get $r \leq k / s$. But for $s \leq r \leq k / s$, by using $(2.2)$, we obtain

$$
|K+K| \geq(4-2 / r) k-(2 r-1) \geq(4-2 / s) k-(2 s-1),
$$

which contradicts (1.2).

We deduce that $r \leq s-1$ and therefore there exist $s-1$ parallel lines which cover the set $K$. This completes the proof.

REMARK. In Theorem A, we may take the following value for $k_{0}(s)$ :

$$
k_{0}(s)=\frac{4 s-2}{\delta(s)}=16(s-1) s(2 s-1)=O\left(s^{3}\right) \text {. }
$$

We end this section by giving an example which shows that Theorem A cannot be improved by increasing the upper bound of $|2 K|$.

ExAmple A. Put $\mathbb{K}=\mathbb{K}_{1} \cup \ldots \cup \mathbb{K}_{s}$ with $\mathbb{K}_{i}=\{(t, i): t=1, \ldots, x\}$. It is clear that if $x>s$, then $\mathbb{K}$ does not lie on $s-1$ parallel lines,

$$
k=|\mathbb{K}|=s x \quad \text { and } \quad|2 \mathbb{K}|=(2 s-1)(2 x-1)=(4-2 / s)|\mathbb{K}|-(2 s-1) .
$$

3. The precise structure of $K \subseteq \mathbb{Z}^{2}$ for $s=3$. We first prove Theorem B. We assume that $K$ lies on the lines $\left\{x_{2}=0\right\}$ and $\left\{x_{2}=1\right\}$. Let the set of abscissae for $x_{2}=0$ and $x_{2}=1$ be equal to $\left\{a_{0}, \ldots, a_{m-1}\right\}$ and $\left\{b_{0}, \ldots, b_{n-1}\right\}$, respectively, $m+n=k$. After a suitable affine isomorphism we may assume that $a_{0}=0, b_{0}=a_{m-1}$ and the greatest common divisor of $\left\{a_{0}, \ldots, a_{m-1}, b_{1}, \ldots, b_{n-1}\right\}$ is equal to 1 . We project the set $K$ onto the line $\left\{x_{2}=0\right\}$ and parallel to the line defined by the points $\left(b_{0}, 1\right)$ and $\left(a_{m-1}, 0\right)$. We recall Freiman's definition [F1], p. 27, for the particular case of a set of lattice points. For some fixed $\kappa$, let $r$ be the number of points in $K$ having the first coordinate $\kappa$, say $\left(\kappa, u_{1}\right), \ldots,\left(\kappa, u_{r}\right)$. Instead of these $r$ points we 
choose the points $(\kappa, 0),(\kappa, 1), \ldots,(\kappa, r-1)$. This process is performed for all fixed $\kappa$ with $r \geq 1$. The set $M$ so obtained is called the projection of the set $K$ onto the line $\left\{x_{2}=0\right\}$.

In our case, we obtain a set $M=M_{1} \cup M_{2}$ where $M_{1}$ is such that the ordinates of its points are all zero, while the set of abscissae is

$$
\left\{a_{0}, \ldots, a_{m-1} ; b_{1}, \ldots, b_{n-1}\right\}
$$

and the set $M_{2}$ consists of the single point $\left(b_{0}, 1\right)$. Using Theorem 1.16 of [F1], p. 27, we get

$$
|2 K| \geq|2 M|=\left|2 M_{1}\right|+(k-1)+1=\left|2 M_{1}\right|+k,
$$

and so

$$
\left|2 M_{1}\right| \leq|2 K|-k<3 k-6=3\left|M_{1}\right|-3 .
$$

Upon recalling that the greatest common divisor of $M_{1}$ is one, Theorem 1.1 therefore yields the conclusion

$$
b_{n-1}-a_{0} \leq\left|2 M_{1}\right|-k+1 \leq|2 K|-2 k+1 .
$$

The proof of Theorem B is now complete.

For a nonempty finite set $X=\left\{x_{1}<\ldots<x_{n}\right\} \subseteq \mathbb{Z}$ we denote by $d(X)$ the greatest common divisor of $\left\{x_{i}-x_{1}: 1 \leq i \leq n\right\}$, by $\ell(X)=x_{n}-x_{1}$ the length of $X$ and by $h_{X}=\ell(X)-|X|+1$ the number of holes in $X$. The assertion of Theorem $B$ may be reworded as follows:

Theorem $\mathrm{B}^{*}$. Let $K$ be a finite subset of $\mathbb{Z}^{2}$ which lies on the lines $\left\{x_{2}=0\right\}$ and $\left\{x_{2}=1\right\}$. Let the sets of abscissae for $x_{2}=0$ and $x_{2}=1$ be $A$ and $B$, respectively.

(a) If $\ell(A)+\ell(B) \leq 2|K|-5$, then $(d(A), d(B))=1$ and

$$
|K+K| \geq 3|K|-3+h_{A}+h_{B}=2|K|-1+\ell(A)+\ell(B) .
$$

(b) If $\ell(A)+\ell(B) \geq 2|K|-4$ and $(d(A), d(B))=1$, then

$$
|K+K| \geq 4|K|-6 \text {. }
$$

Proof. Note first that $d=(d(A), d(B)) \geq 2$ implies $\ell(A)+\ell(B) \geq$ $d(|A|-1)+d(|B|-1) \geq 2(|A|+|B|-2)=2|K|-4$.

(a) We assume $\ell(A)+\ell(B) \leq 2|K|-5$.

If $|2 K| \geq 4|K|-6$, then inequality (3.1) is true because $2|K|-1+\ell(A)+$ $\ell(B) \leq 4|K|-6 \leq|2 K|$. get

Suppose $|2 K|<4|K|-6$. By using $(d(A), d(B))=1$ and Theorem B we $|2 K|=l_{1}+l_{2}+2 k-3 \geq(\ell(A)+1)+(\ell(B)+1)+2 k-3=2|K|-1+\ell(A)+\ell(B)$. 
(b) We assume $\ell(A)+\ell(B) \geq 2|K|-4$.

Suppose $|2 K|<4|K|-6$. By using $(d(A), d(B))=1$ and Theorem B we obtain

$$
|2 K|-2 k+3=l_{1}+l_{2} \geq \ell(A)+1+\ell(B)+1,
$$

and so $\ell(A)+\ell(B) \leq|2 K|-2 k+1<2 k-5$, which contradicts the assumption of (b).

The proof of Theorem $\mathrm{B}^{*}$ is complete.

We now present some examples:

EXAMPle B.1. An investigation of the set (3.3) $K_{0}^{\prime}=\{(0,0),(1,0), \ldots,(m-2,0),(x, 0) ;(0,1),(1,1), \ldots,(n-1,1)\}$, where $x>2 \max \{m, n\}$, gives $\left|K_{0}^{\prime}+K_{0}^{\prime}\right|=4\left|K_{0}^{\prime}\right|-6$ and shows that inequality (3.2) cannot be improved.

ExAmple B.2. The set

$$
K_{0}^{\prime \prime}=\{(0,0),(1,0), \ldots,(m-3,0),(x, 0) ;(0,1)\},
$$

where $m-3<x<2 m-6$, gives $h_{A}=x-m+2, h_{B}=0$ and

$$
\left|K_{0}^{\prime \prime}+K_{0}^{\prime \prime}\right|=x+2 m-1=3\left|K_{0}^{\prime \prime}\right|-3+h_{A}+h_{B} .
$$

This shows that inequality (3.1) is sharp.

4. On the precise structure of $K \subseteq \mathbb{Z}^{2}$ for $s=4$. In this section we prove Theorem C. Assume $k_{0}=k_{0}(4)=1344$. Using (2.25), Theorem A and inequality $(2.2)$ we get

$$
\frac{10}{3} k-5 \leq|K+K|<3.5 k-7
$$

and also that $K$ is decomposable into three sets $\mathcal{A}, \mathcal{B}, \mathcal{C}$ which lie on three parallel lines $\left\{x_{2}=u\right\},\left\{x_{2}=v\right\},\left\{x_{2}=w\right\}$, respectively, where $u<v<w$.

If $2 v \neq u+w$ then $2 \mathcal{B} \cap(\mathcal{A}+\mathcal{C})=\emptyset$. Therefore

$$
|K+K| \geq|2 \mathcal{A}|+|\mathcal{A}+\mathcal{B}|+|2 \mathcal{B}|+|\mathcal{B}+\mathcal{C}|+|2 \mathcal{C}|+|\mathcal{A}+\mathcal{C}| \geq 4|K|-6,
$$

which contradicts (4.1). Hence, we may assume that $K$ lies on the lines: $\left\{x_{2}=0\right\},\left\{x_{2}=1\right\},\left\{x_{2}=2\right\}$. Let the sets of abscissae of $K$ for $x_{2}=0$, $x_{2}=1, x_{3}=2$ be $A, B, C$, respectively.

If $\mathcal{B} 2 \cap(\mathcal{A}+\mathcal{C})=\emptyset$, then

$$
\begin{aligned}
|K+K| & \geq|2 A|+|A+B|+(|A+C|+|B 2|)+|B+C|+|2 C| \\
& =4|A|+3|B|+4|C|-5 .
\end{aligned}
$$

But

$|K+K| \geq|2 A|+|A+B|+|2 B|+|B+C|+|2 C|=3|A|+4|B|+3|C|-5$. 
By taking the arithmetic mean we get

$$
|K+K| \geq 3.5|K|-5,
$$

which contradicts (4.1).

Therefore we suppose that $\mathcal{B} 2 \cap(\mathcal{A}+\mathcal{C})$ is a nonempty set. After a suitable affine isomorphism of $\mathbb{R}^{2}$, we find $t \in \mathbb{Z}$ such that

$$
(t, 0) \in \mathcal{A}, \quad(t, 1) \in \mathcal{B}, \quad(t, 2) \in \mathcal{C} .
$$

We project the set $K$ onto the line $\left\{x_{2}=0\right\}$ (see Section 3 for the definition). The projection $M$ is decomposable into three subsets $\mathcal{M}_{1}, \mathcal{M}_{2}, \mathcal{M}_{3}$, which lie on three parallel lines $\left\{x_{2}=0\right\},\left\{x_{2}=1\right\},\left\{x_{2}=2\right\}$. Let the sets of abscissae of $M$ for $x_{2}=0, x_{2}=1, x_{2}=2$, respectively, be equal to

$$
M_{1}=\left\{a_{0}, \ldots, a_{m-1}\right\} \supseteq M_{2}=\left\{b_{0}, \ldots, b_{n-1}\right\} \supseteq M_{3}=\left\{c_{0}, \ldots, c_{p-1}\right\} .
$$

Here $m+n+p=k$ and $p \geq 1$, by (4.2). Without loss of generality, we can assume that the greatest common divisor of $\left\{a_{1}-a_{0}, \ldots, a_{m-1}-a_{0}\right\}$ is 1 , and $a_{0}=0$. Let us define $l_{1}, l_{2}, l_{3}$ by

$$
l_{1}=1+\max (A), \quad l_{2}=1+\max (B), \quad l_{3}=1+\max (C) .
$$

Theorem 1.16 of [F1], p. 27, states that

$$
|2 M| \leq|2 K| \text {. }
$$

Moreover, it is obvious that $p=|A \cap B \cap C|$,

$$
A \cup B \cup C=\left\{a_{0}, \ldots, a_{m}\right\},
$$

$\min (A \cup B \cup C)=a_{0}=0, \quad \max (A \cup B \cup C)=a_{m-1}=\max \left(l_{1}, l_{2}, l_{3}\right)-1$

and $K \subseteq K_{0}$, where

$$
\begin{aligned}
K_{0}= & \left\{(i, 0): i=0, \ldots, l_{1}-1\right\} \cup\left\{(j, 1): j=0, \ldots, l_{2}-1\right\} \\
& \cup\left\{(s, 2): s=0, \ldots, l_{3}-1\right\} .
\end{aligned}
$$

Therefore, $\max \left(l_{1}, l_{2}, l_{3}\right)-1=a_{m-1}-a_{0}$ is exactly the length of $M_{1}=$ $M \cap\left\{x_{2}=0\right\}$.

We now estimate the cardinality of $M_{1}+M_{2}$, using the following result from $[\mathrm{F} 2]$.

Theorem 4.1. Let $M$ and $N$ be finite sets of nonnegative integers, $0 \in$ $M, 0 \in N$.

(a) If $\max (\ell(M), \ell(N))=\ell(M) \leq|M|+|N|-3$, then $|M+N| \geq$ $\ell(M)+|N|$.

(b) If $\max (\ell(M), \ell(N)) \geq|M|+|N|-2$ and $d(M \cup N)=1$, then

$$
|M+N| \geq|M|+|N|-3+\min (|M|,|N|) .
$$

This is a generalization of Theorem 1.1 to the case of distinct summands. For some improvements of Theorem 4.1 see $[\mathrm{L}-\mathrm{S}]$ and $[\mathrm{S}]$. 
If $a_{m-1} \geq m+n-2$, then $\left|M_{1}+M_{2}\right| \geq m+n-3+n=m+2 n-3$. It follows that

$$
\begin{aligned}
|2 K| & \geq|2 M| \geq\left|2 M_{1}\right|+\left|M_{1}+M_{2}\right|+\left|M_{1}+M_{3}\right|+\left|M_{2}+M_{3}\right|+\left|2 M_{3}\right| \\
& \geq(2 m-1)+(m+2 n-3)+(m+p-1)+(n+p-1)+(2 p-1) \\
& =4 m+3 n+4 p-7 \geq 3.5 k-7,
\end{aligned}
$$

since $m \geq n$, which contradicts (4.1).

Thus, we assume that $a_{m-1} \leq m+n-3$; it follows that $\left|M_{1}+M_{2}\right| \geq$ $a_{m-1}+n$, and so we obtain

$$
\begin{aligned}
|2 K| & \geq|2 M| \\
& \geq\left|2 M_{1}\right|+\left(a_{m-1}+n\right)+(m+p-1)+(n+p-1)+(2 p-1) \\
& =\left|2 M_{1}\right|+a_{m-1}+m+2 n+4 p-3 .
\end{aligned}
$$

Suppose $\left|2 M_{1}\right| \geq 3 m-3$. Using (4.4) and $a_{m-1} \geq m-1$ we obtain $|2 K| \geq$ $5 m+2 n+4 p-7 \geq 3.5|K|-7$, which contradicts (4.1). We conclude that

$$
\left|2 M_{1}\right|<3 m-3 \text {. }
$$

However, the greatest common divisor of $M_{1}$ is one and therefore by Theorem 1.1 we obtain

$$
1+a_{m-1} \leq\left|2 M_{1}\right|-m+1 \leq|2 K|-\left(2 m+2 n+4 p+a_{m-1}-3\right)+1
$$

This yields the conclusion

$$
\begin{aligned}
\ell\left(M_{1}\right)=a_{m-1} & \leq \frac{1}{2}|2 K|-(m+n+2 p)+1.5=\frac{1}{2}|2 K|-k-p+1.5 \\
& \leq \frac{1}{2}|2 K|-k+0.5
\end{aligned}
$$

that is,

$$
\begin{aligned}
|2 K| & \geq(2 k-1)+2 \ell\left(M_{1}\right)+2(p-1)=(4 k-5)+2 h_{M_{1}}-2 n \\
& \geq(2 k-1)+2 \ell\left(M_{1}\right)=(2 k-1)+2\left[\max \left(l_{1}, l_{2}, l_{3}\right)-1\right] .
\end{aligned}
$$

The proof of Theorem $\mathrm{C}$ is now complete.

We end this section by giving a direct version of Theorem C. We use the same notation as above.

First situation. In the proof of Theorem $\mathrm{C}$ we noticed that if $K$ does not lie on three equidistant lines, or if $\mathcal{B} 2 \cap(\mathcal{A}+\mathcal{C})=\emptyset$, then $|2 K| \geq 3.5|K|-7$.

Second situation. Suppose that $K$ lies on the lines $\left\{x_{2}=0\right\},\left\{x_{2}=1\right\}$, $\left\{x_{2}=2\right\}$, and $\mathcal{B} 2 \cap(\mathcal{A}+\mathcal{C})$ is a nonempty set. After a suitable affine isomorphism of $\mathbb{R}^{2}$, we assume that there are at least three points in $K$ with the same abscissa. Denote by $L=L(K)=\ell(A \cup B \cup C)$ the length of $K$ and by $D=D(K)=d(A \cup B \cup C)$ the greatest common divisor of $K$. Theorem $\mathrm{C}$ may be reworded as follows: 
Theorem $\mathrm{C}^{*}$. Let $K$ be a finite subset of $\mathbb{Z}^{2}$ which lies on the lines $\left\{x_{2}=0\right\},\left\{x_{2}=1\right\}$ and $\left\{x_{2}=2\right\}$. Let the sets of abscissae for $x_{2}=0$, $x_{2}=1$ and $x_{2}=2$ be $A, B$ and $C$, respectively. Suppose that $0 \in A \cap B \cap C$.

(a) If $L / D<\frac{3}{4} k-3$, then

$$
|K+K| \geq(2|K|-1)+2 L / D .
$$

(b) If $L / D \geq \frac{3}{4} k-3$, then

$$
|K+K| \geq 3.5|K|-7 .
$$

P r o of. (a) We distinguish two cases. If $|2 K| \geq 3.5 k-7$, then inequality (4.8) is a consequence of $L / D<\frac{3}{4} k-3$.

Suppose now that

$$
|2 K|<3.5 k-7 \text {. }
$$

Note that $0 \in A \cap B \cap C$ implies $A \cup B \cup C \subseteq D \mathbb{Z}$. Put $K^{\prime}=\{(x / D, y)$ : $(x, y) \in K\}$ and denote by $M^{\prime}$ the projection of $K^{\prime}$ on the line $\left\{x_{2}=0\right\}$. The set of abscissae of $M^{\prime} \cap\left\{x_{2}=0\right\}$ has length $L / D$. Therefore, inequality (4.7) gives

$$
|2 K|=\left|2 K^{\prime}\right| \geq\left(2\left|K^{\prime}\right|-1\right)+2 L / D=(2|K|-1)+2 L / D,
$$

and inequality $(4.8)$ is proved.

(b) Assume $L / D \geq \frac{3}{4} k-3$ and $|2 K|<3.5 k-7$. Apply Theorem C to the set $K^{\prime}$ defined above. We find that (4.11) is true. Thus, $L / D \leq$ $\frac{1}{2}(|2 K|-2 k+1)<\frac{1}{2}(3.5 k-7-2 k+1)=\frac{3}{4} k-3$, which contradicts the assumption of (b). The proof of Theorem $\mathrm{C}^{*}$ is complete.

Example A, for $s=4$, shows that Theorem $\mathrm{C}$ cannot be improved by increasing the upper bound of $|2 K|$.

EXAMPLE C. An investigation of the set

$$
\begin{aligned}
K_{0}=\{(0,0),(1,0), \ldots,(2 l-2,0),(x, 0) ; \\
(0,1),(1,1), \ldots,(l-2,1) ;(0,2)\}
\end{aligned}
$$

where $2 l-1 \leq x \leq 3 l-4$, gives

$$
l_{1}=x+1, \quad l_{2}=l-1, \quad l_{3}=1, \quad\left|K_{0}\right|=3 l, \quad \max \left(l_{1}, l_{2}, l_{3}\right)=x+1
$$

and

$$
L=L\left(K_{0}\right)=x, \quad D=D\left(K_{0}\right)=1 .
$$

It is easy to verify that if $2 l-1 \leq x \leq 3 l-4$, then $\left|2 K_{1}\right|=x+2 l,\left|K_{1}+K_{2}\right|=$ $x+l-1,\left|\left(K_{1}+K_{3}\right) \cup 2 K_{2}\right|=\left|K_{1}+K_{3}\right|=2 l,\left|K_{2}+K_{3}\right|=l-1,\left|2 K_{3}\right|=1$. Thus

$$
\left|2 K_{0}\right|=\left(2\left|K_{0}\right|-1\right)+2 x=\left(2\left|K_{0}\right|-1\right)+2 L / D .
$$


Therefore, inequality (4.8) cannot be improved. Moreover, if $x=\frac{3}{4}\left|K_{0}\right|-3=$ $\frac{9}{4} l-3$, then

$$
\left|2 K_{0}\right|=3.5\left|K_{0}\right|-7
$$

Thus, inequality (4.9) is also sharp.

Acknowledgments. Prof. G. A. Freiman directed my attention to this problem and I would like to thank him for his guidance and help.

I am indebted to Dr. Y. Bilu (Forschungsinstitut für Mathematik, Zürich) for his remarks concerning the first version of the paper presented at the conference on Structure Theory of Set Addition, CIRM, Marseille, France, 1993. I also profited much from discussions with Dr. V. F. Lev (University of Georgia, Athens, USA). Finally, I thank the referee for his very careful work.

\section{References}

[B] Y. Bilu, Structure of sets with small sumsets, Mathématiques Stochastiques, Univ. Bordeaux 2, Preprint 94-10, Bordeaux, 1994.

[F1] G. A. Freiman, Foundations of a Structural Theory of Set Addition, Transl. Math. Monographs 37, Amer. Math. Soc., Providence, R.I., 1973.

[F2] - Inverse problems in additive number theory VI. On the addition of finite sets III, Izv. Vyssh. Uchebn. Zaved. Mat. 1962, no. 3 (28), 151-157 (in Russian).

[F3] - What is the structure of $K$ if $K+K$ is small?, in: Lecture Notes in Math. 1240, Springer, 1987, New York, 109-134.

[L-S] V. F. Lev and P. Y. Smeliansky, On addition of two distinct sets of integers, Acta Arith. 70 (1995), 85-91.

[R] I. Z. Ruzsa, Generalized arithmetical progressions and sumsets, Acta Math. Hungar. 65 (1994), 379-388.

[S] Y. Stanchescu, On addition of two distinct sets of integers, Acta Arith. 75 (1996), 191-194.

School of Mathematical Sciences

Raymond and Beverly Sackler Faculty of Exact Sciences

Tel-Aviv University

Tel-Aviv, Israel

E-mail: ionut@math.tau.ac.il 